\title{
Consulta de enfermería interdisciplinar de enfermedad renal crónica avanzada: apuntes para un modelo integral de cuidados
}

\author{
Helena García Llana ${ }^{1 *}$, Rocío Rodríguez Rey ${ }^{1 * *}$, Filo Trocoli González ${ }^{2 *}$, Olga Celadilla Díez ${ }^{2 *}$, Solmar \\ Rodríguez Gutiérrez ${ }^{1 * *}$, María Arranz Sánchez ${ }^{1 * * *}$, Auxiliadora Bajo Rubio ${ }^{3 *}$, Rafael Sánchez Villanueva ${ }^{3 *}$, \\ Gloria del Peso Gilsanz ${ }^{3 *}$, Elena González García ${ }^{3 *}$, Rafael Selgas Gutiérrez ${ }^{3 *}$
}

${ }^{1}$ Psicólogo, ${ }^{2}$ Enfermero, ${ }^{3}$ Nefrólogo. * Servicio de Nefrología. Hospital Universitario La Paz. IdiPAZ, ** Facultad de Psicología. Universidad Autónoma de Madrid. *** Facultad de Psicología. Universidad Complutense de Madrid

\section{Sr. Director}

La consulta de enfermería de Enfermedad Renal Crónica Avanzada (ERCA) constituye un escenario especialmente complejo ${ }^{(1,2)}$ tanto para el paciente como para los profesionales. Por este motivo, y con el objetivo principal de ofrecer apoyo al personal de enfermería durante estas consultas, en la Unidad de ERCA del Servicio de Nefrología del Hospital Universitario La Paz, se encuentra dentro del protocolo de actuación la posibilidad de que el psicólogo este físicamente presente en esta consulta. Esta modalidad de actuación está en línea con la evidencia de que un equipo interdisciplinar es necesario para mejorar la comprensión de la enfermedad renal, y con ello la calidad de vida relacionada con la salud (CVRS) de los pacientes con ERCA ${ }^{(3)}$.

Este protocolo ha sido publicado como carta al director en la revista Nefrología ${ }^{(4)}$, aunque en la presente versión hacemos énfasis en aspectos diferenciales, principalmente en las funciones específicas de la enfermería en la consulta de ERCA y la definición del perfil de paciente ante el cual activar este protocolo. El objetivo de promover la difusión del mismo en las distintas sociedades científicas de la especialidad es recordarnos que en el abordaje integral del enfermo renal es mucho más lo que nos une que lo que nos separa.

\section{Correspondencia:}

Helena García Llana

Servicio de Nefrología. Hospital General

Hospital Universitario La Paz- IdiPAZ

Paseo de la Castellana, 261.28046 Madrid

E-mail: helenagllana@hotmail.com
Funciones de la enfermería y del psicólogo en la consulta ERCA de enfermería

La principal función del psicólogo en la consulta de ERCA de enfermería es apoyar a dicho profesional en el proceso de comunicación, apoyo emocional y toma de decisiones compartida (TDC), siendo la enfermería quien, normalmente, asume el papel de dirigir y marcar el ritmo de la consulta. En la Tabla 1 se indican con mayor detalle las funciones de ambos profesionales en esta consulta especializada.

\section{Protocolo conjunto enfermera-psicólogo en consulta de ERCA}

Consensuar con el equipo asistencial ante qué perfil de paciente es conveniente que el psicólogo se encuentre presente en la consulta de ERCA de enfermería.

Esta modalidad de escenario interdisciplinar sólo se realiza ante determinados perfiles de pacientes donde la intervención simultánea pretende facilitar la adaptación al futuro tratamiento al menor coste emocional posible. El perfil de paciente de difícil manejo ha sido descrito en el contexto de la hemodiálisis ${ }^{(5)}$, pero en nuestra búsqueda no hemos encontrado literatura específica en el ámbito del paciente con ERCA. En este estadío concreto, los pacientes de alta complejidad psicológica son aquellos donde se ha identificado, previamente por el nefrólogo responsable y/o la enfermera una o varias condiciones que puedan dificultar el proceso comunicativo, así como la posterior TDC y la adaptación al tratamiento renal sustitutivo (TRS). Algunas de las condiciones más frecuentes que nos hemos encontrado son:

- Historia psiquiátrica activa.

- Negación intensa, persistente y desadaptativa ${ }^{(6)}$ de la ERCA y sus tratamientos en el paciente y/o familia. 
Funciones del profesional de enfermería en consulta de ERCA (Adaptado de Bardón-Otero y Marti i Monros, 2008)(1)

Conservar la función renal el mayor tiempo posible y disminuir la comorbilidad: potenciar los autocuidados y la autonomía del paciente tratando de optimizar su calidad de vida en el período pre elección de TRS.

Valoración inicial del paciente: estilo de afrontamiento, grado de conocimiento de la enfermedad y tratamientos, estilo de vida y/o factores de riesgo cardiovascular, adhesión terapéutica y apoyo social disponible.

PROCESO EDUCATIVO-MOTIVACIONAL DE ELECCIÓN DE TRS Planificación de cuidados con intervención educativa personalizada según los objetivos a alcanzar: proceso comunicativo sobre distintas opciones de TRS y pautas de toma de medicación y dieta.

Ofrecer apoyo emocional* a lo largo del proceso de elección sobre TRS.

Realizar una exploración de los valores y estilo de vida del paciente y familia para la TDC.

Planificar la entrada al TRS elegido: coordinar la planificación del Acceso Vascular y/o catéter peritoneal, evitando el uso de accesos temporales.

Evaluación de resultados y planteamiento de reintervención educativa.
Funciones del psicólogo de apoyo a enfermería en consulta de ERCA (Adaptado de García-Llana et al, 2013)(4)

Promover la adhesión al tratamiento como variable facilitadora de conservación de la función renal.

Apoyo a enfermería en la valoración inicial del paciente prestando especial tención a las necesidades y recursos psicológicos del paciente y su familia.

A. Evaluar el estado emocional del paciente durante el proceso de transmisión de información a través de la observación del lenguaje no verbal y de la realización de preguntas abiertas y focalizadas.

B. Detectar posibles dificultades en la comprensión de la información por parte del paciente, clarificar contenidos y favorecer que el paciente exponga sus dudas.

C. Realizar una intervención en crisis si se presentan reacciones emocionales intensas de difícil manejo en consulta.

Reforzar al profesional de enfermería en la facilitación del apoyo emocional al paciente.

Apoyar al profesional de enfermería en exploración de valores y del estilo de vida del paciente.

Ofrecer la disponibilidad de una evaluación en profundidad y posterior apoyo psicológico al paciente y/o familia en caso de estar indicado.

Apoyo a enfermería en el proceso de seguimiento del paciente y reevaluación de apoyo psicológico.

Evaluación de resultados (estado emocional, adhesión al tratamiento, calidad de vida etc.)

Tabla 1. Funciones del profesional de enfermería y del psicólogo en la consulta de enfermería de ERCA.

Nota: * Se entiende por dar apoyo emocional la creación de una relación terapéutica en la que el paciente no se sienta juzgado, sino entendido y ayudado para poder confrontar, desde el respeto, sus temores, deseos y necesidades.

- Reacciones emocionales intensas en el paciente $y / 0$ familia.

- Dificultades manifiestas de autocuidado y/o adhesión a los tratamientos.

- Situación de riesgo social y/o desestructuración familiar.

- Dificultades previas en la relación con profesionales sanitarios.

\section{Activación del protocolo interdisciplinar.}

1. Acogida y presentación de los profesionales y del paciente-familia. Es imprescindible la presentación del psicólogo como parte del equipo interdisciplinar, ya que, la presencia de este profesional en la consulta puede extrañar al paciente, e incluso provocar resistencias. Esta introducción la lidera el profesional de enfermería, que, además, con frecuencia tendrá un conocimiento previo más profundo del paciente.

2. Explicitar el objetivo principal de la consulta (información de las diferentes modalidades de TRS para inicio del proceso de TDC sobre la base de los valores del paciente). Esta parte la continúa liderando el profesional de enfermería.
3. Indicar que el proceso de información y TDC es un proceso gradual, y no un acto único, de modo que podemos detenernos y continuar otro día. Esta información es explicitada por el psicólogo.

4. Explicar las distintas técnicas de TRS mediante el apoyo de objetos físicos y soportes multimedia. Esta parte central de la consulta será realizada por el profesional de enfermería, mientras el psicólogo estará atento a las claves de lenguaje no verbal del paciente, de cara a valorar si su estado emocional es el adecuado para continuar con el proceso comunicativo.

5. Explorar si el paciente o la familia tienen dudas y resolverlas facilitando la comunicación asertiva entre paciente-familia-equipo asistencial. Esta parte es realizada conjuntamente por el profesional de enfermería y psicólogo.

6. Explorar el mundo de valores del paciente y el estilo de vida conjuntamente.

7. Apoyo en el inicio de la TDC, por parte de ambos profesionales, mediante un proceso deliberativo ${ }^{(7)}$ basado en los valores del paciente.

8. Resumen, normalización de reacciones emocionales y ofrecimiento de seguimiento por parte del psicólogo. 
9. Planificación y seguimiento por parte del profesional de enfermería y del psicólogo en caso de que sea necesario. Existe la posibilidad de realizar más consultas conjuntas si se precisa.

10. Puesta en común de la decisión del paciente con el nefrólogo responsable.

\section{Reflexión final: condiciones previas y dirección de futuro}

La presencia del psicólogo en la consulta de enfermería de ERCA no es un hecho habitual, y requiere formación por parte de ambos profesionales. Es imprescindible que el psicólogo conozca los aspectos médicos básicos y de cuidados de enfermería de un paciente en ERCA para que su apoyo sea útil en este proceso. Asimismo, es imprescindible que el profesional de enfermería reciba formación acerca de claves de comunicación terapéutica.

La modalidad de trabajo interdisciplinar que hemos presentado no es cerrada ni exclusiva, sino que está abierta a otras posibilidades y escenarios de acción. Por un lado, la intervención conjunta de estos dos profesionales no tiene por qué limitarse al contexto de la consulta de ERCA de enfermería, sino que puede realizarse en situaciones de hospitalización, o en la sala de hemodiálisis con el objetivo de promover autocuidados. Por otro, la colaboración interdisciplinar no es exclusiva del binomio enfermería-psicólogo, sino que puede producirse entre otros profesionales, como dietista-psicólogo, o médico-psicólogo, por citar algunos ejemplos.

Estas alianzas interdisciplinares que incorporan la figura del psicólogo nos permiten maximizar los resultados en salud y en satisfacción en el paciente y en su familia dentro del ámbito de la cronicidad, y se encuentran en línea con la idea de que la nefrología del siglo XXI debe considerar la creación de equipos interdisciplinares en el tratamiento de la ERCA, así como un abordaje integral biopsicosocial de la enfermedad ${ }^{(8)}$.

Recibido: 22 noviembre 2012

Revisado: 10 diciembre 2012

Modificado: 20 Enero 2013

Aceptado: 30 enero 2013

\section{Bibliografía}

1. Bardón-Otero E, Marti i Monros A. Enfermería en la consulta de enfermedad renal crónica avanzada (ERCA) Nefrología. 2008; Supl. 3, 53-56.

2. Orte-Martínez L, Barril-Cuadrado G. Unidad de enfermedad renal crónica avanzada (ERCA). Concepto de una unidad multidisciplinaria. Objetivos de la consulta de ERCA. Nefrología. 2008; Supl 3, 49-52.

3. Fox C, Kohn LS. The importance of patient education in the treatment of chronic kidney disease. Kidney International. 2008; 74, 1114-5.

4. García-Llana $H$, Rodríguez-Rey $R$, Celadilla 0 , Bajo A, Sánchez-Villanueva R, del Peso G, González E, Trocoli $F$, Selgas R. Abordaje interdisciplinar enfermera-psicólogo en la consulta de enfermedad renal crónica avanzada: objetivos y protocolo. Nefrología. 2013; 33(1):139-140.

5. Levine DZ. What is our duty to a "hateful" patient? Differing approaches to a disruptive dyalisis patient.

6. Arranz P, Barbero, J; Barreto P, Bayés R. Intervención emocional en cuidados paliativos. Modelo y protocolos. Barcelona: Ariel; 2003.

7. Gracia D. La deliberación moral: el método de la ética clínica. Med Clin (Barc) 2001; 117 (1): 16-7.

8. Santacruz PL, Rangel ME, Navas N, Bolívar Z. La visión integradora biopsicosocial como estrategia ante el paciente con enfermedad renal crónica. Requisito contemporáneo. Nefrología 2005, 26 (5): 635-636. 